\title{
Estudo longitudinal da população materno-infantil da região urbana do Sul do Brasil, 1993: aspectos metodológicos e resultados preliminares
}

\section{Longitudinal study of the mother and child population in an urban re- gion of southern Brazil, 1993: methodological aspects and preliminary results}

\author{
Cesar G. Victora, Fernando C. Barros, Ricardo Halpern, Ana M. B. Menezes, Bernardo L. \\ Horta, Elaine Tomasi, Elizabeth Weiderpass, Juraci A. Cesar, Maria Teresa Olinto, Paula R. \\ V. Guimarães, Maria del Mar Garcia e J. Patrick Vaughan \\ Departamento de Medicina Social, Materno-Infantil e de Clínica Médica. Faculdade de Medicina da \\ Universidade Federal de Pelotas. Pelotas, RS- Brasil (C.G.V.; F.C.B; R.H.; A.M.M.; B.L.H; E.T.; E.W.; \\ P.R.V.G.); Departamento Materno-Infantil da Universidade Federal do Rio Grande. Rio Grande, RS- \\ Brasil (J.A.C.); Escuela Andaluza de Salud Publica, Granada, Espanha (M.M.G.); Department of Public \\ Health and Policy, London School of Hygiene and Tropical Medicine, Reino Unido (J.P.V.)
}

\begin{abstract}
Resumo
Em 1982, todos os nascimentos ocorridos na cidade de Pelotas, RS - Brasil, foram estudados e essas crianças foram acompanhadas prospectivamente durante os primeiros anos de vida. Em 1993, repetiu-se o estudo com metodologia similar, com a finalidade de avaliar as eventuais mudanças no nível de saúde maternoinfantil, ocorridas durante esses onze anos. Todas as cinco maternidades da cidade foram visitadas diariamente e os 5.304 nascimentos ocorridos foram incluídos no estudo. As crianças foram pesadas e medidas, sendo sua idade gestacional avaliada através do método de Dubowitz. As mães foram examinadas e entrevistadas sobre um grande número de fatores de risco. A mortalidade dessas crianças foi monitorizada por visitas regulares a hospitais, cemitérios e registros de óbito, e todas as internações hospitalares foram acompanhadas. Dois estudos aninhados de casos e controles foram realizados para investigar fatores de risco para mortalidade e hospitalizações. Uma amostra sistemática de 655 crianças foram examinadas em casa com um e três meses de idade, e essas mesmas crianças, acrescidas de outras 805 (que incluíram todos os recém-nascidos de baixo peso) foram também acompanhadas aos seis e doze meses de idade. O desenvolvimento psicomotor dessas amostras foi também avaliado. As perdas de acompanhamento aos doze meses foram de apenas 6,6\%. Em relação aos dados de 1982, a pesquisa de 1993 mostrou redução de cerca de $30 \%$ na mortalidade perinatal e de quase $50 \%$ na mortalidade infantil, assim como aumento de um mês na duração mediana da amamentação. Por outro lado, não houve qualquer alteração nas prevalências de baixo peso ao nascer e de déficit de comprimento/idade aos doze meses. $\mathrm{O}$ artigo a que se refere este resumo descreve a metodologia do estudo, ao qual se segue uma série de outras publicações.
\end{abstract}

Saúde materno-infantil. Estudos longitudinais. Pesquisa, métodos.

Correspondência para/Correspondence to: Cesar G. Victora - Departamento de Medicina Social. Faculdade de Medicina da Universidade Federal de Pelotas. Caixa Postal 464 - 96001-970 - Pelotas, RS - Brasil. Fax: (0532) 71-2442

Recebido em 12.7.1995. Aprovado em 6.11.1995. 


\begin{abstract}
All babies born in the hospitals of the city of Pelotas, Brazil, in 1982 were studied soon after delivery and followed up prospectively during the first years of their lives. In 1993, this study was repeated with a similar methodology, with the aim of assessing eventual changes in the level of maternal and child health. All five maternity hospitals in the city were visited daily and the 5,304 babies born included in the study. They were weighed and measured, and their gestational age was assessed using the Dubowitz method. Their mothers were examined and interviewed regarding a large number of risk factors. The mortality of these children was studied through the surveillance of all hospitals, cemeteries and death registries, and all hospital admissions were also recorded. Two nested case-control studies were carried out to assess risk factors for mortality and hospital morbidity. A systematic sample of 655 children were examined at home at one and three months of age, and these infants, as well as another sample of 805 children including all lowbirthweight babies were also examined at the ages of six and twelve months. Their psychomotor development was also assessed. Losses to follow-up were only $6.6 \%$ at twelve months. Relative to the 1982 indicators, perinatal mortality fell by about $30 \%$ and infant mortality by almost $50 \%$. The median duration of breastfeeding increased from 3.1 to 4.0 months. On the other hand, there was little change in the prevalences of low birthweight or of length for age at twelve months. The article that refers this abstract describes the methodology of the study and forthcoming publications will present detailed results.
\end{abstract}

Maternal and child health. Longitudinal studies. Research, methods.

\section{INTRODUÇÃO}

Os anos 1980 e 1990 foram caracterizados, no Brasil, por programas de ajuste econômico com importante impacto sobre a qualidade de vida da população, principalmente de seu segmento mais vulnerável, o materno-infantil ${ }^{9}$. Para verificar a magnitude desse impacto sobre as mães e crianças, é necessário avaliar periodicamente suas condições de saúde.

A cidade de Pelotas, RS, apresenta-se em posição privilegiada para este tipo de avaliação, devido a existência de dados históricos confiáveis. Em 1982, todas as crianças nascidas nos hospitais da cidade foram identificadas e acompanhadas prospectivamente durante os primeiros anos de vida ${ }^{1,15}$. Sua morbi-mortalidade, padrões alimentares, crescimento e desenvolvimento psicológico, assim como as condições socioeconômicas sob as quais viviam, foram registradas e analisadas em uma série de publicações.

O estudo de 1982 subsidiou o desenvolvimento de planos de saúde, incluindo o estabelecimento de rede de cuidados primários, a implantação de unidades de cuidados intensivos neonatais e a promoção do aleitamento natural, não apenas na cidade de Pelotas, mas em vários outros municípios brasileiros.
Para avaliar a extensão da transição de saúde na última década e o possível impacto das políticas socioeconômicas, decidiu-se acompanhar uma nova coorte, também constituída de todos os nascimentos hospitalares que ocorreram na cidade no ano de 1993. Os novos resultados obtidos poderão ser comparados com os dados de 1982, propiciando assim a análise das tendências históricas.

A experiência anterior mostrou que as mais importantes alterações na saúde infantil ocorriam durante o primeiro ano de vida, o que orientou o presente estudo a concentrar-se nesta faixa etária. O estudo teve como objetivos principais:

- Medir diversos indicadores de morbimortalidade e de desenvolvimento em todas as crianças nascidas na cidade no ano de 1993, assim como em suas mães.

- Caracterizar essa população em termos socioeconômicos, demográficos, ambientais e quanto à utilização de serviços de saúde.

-Comparar estes resultados com os obtidos em 1982 e interpretar as eventuais modificações à luz das alterações econômicas e nas políticas de saúde.

-Subsidiar o planejamento local de ações de saúde materno-infantil e identificar grupos de risco para intervenções sanitárias. 
$\mathrm{O}$ volumoso banco de dados produzido pelo estudo será útil, ainda, para testar diversas hipóteses recentes sobre determinantes da saúde maternoinfantil. No presente artigo são descritos os principais aspectos metodológicos da pesquisa de 1993 assim como alguns resultados iniciais.

\section{METODOLOGIA}

O estudo longitudinal foi composto de cinco subprojetos, cada qual com metodologia e logística específicas. Os subprojetos incluíram as pesquisas perinatal, de acompanhamento (um, três, seis e doze meses), de mortalidade, de morbidade hospitalar e de desenvolvimento infantil. Paralelamente, outros dois projetos foram realizados para subsidiar a interpretação dos resultados obtidos no estudo longitudinal, quais sejam a análise da evolução do setor saúde, no Município de Pelotas, entre 1982 e 1993, e um estudo etnográfico com o objetivo de avaliar a percepção das mães sobre estas mudanças. Estes dois últimos subprojetos não serão descritos no presente artigo.

O estudo longitudinal teve uma coordenação central (FCB e CGV), tendo cada subprojeto um coordenador de trabalho de campo. Foi envolvida no trabalho equipe multidisciplinar de cerca de 60 pessoas incluindo epidemiologistas, médicos, antropólogos, nutricionistas, estatísticos, alunos de graduação e pós- graduação e pessoal administrativo. O estudo foi financiado pela Comunidade Econômica Européia, pela Fundação de Amparo à Pesquisa do Rio Grande do Sul, pelo Conselho Nacional de Desenvolvimento Científico e Tecnológico e pela Divisão de Diarréia da Organização Mundial da Saúde. Contou-se ainda com a participação da London School of Hygiene and Tropical Medicine (JPV) e da Escuela Andaluza de Salud Publica (MMG).

\section{Metodologia do Subprojeto Perinatal}

Os principais objetivos do subprojeto perinatal incluíram medir as frequiências de baixo peso ao nascer, retardo de crescimento intra-uterino e partos prematuros, assim como estudar o efeito de fatores demográficos, socioeconômicos, ambientais, maternos e assistenciais sobre estes indicadores. Tentou-se recrutar todas as mulheres que tiveram filhos nas cinco maternidade da cidade, de primeiro de janeiro a 31 de dezembro de 1993. Estas foram entrevistadas utilizando-se um questionário padronizado com perguntas sobre condições socioeconômicas da família, dados demográficos, história reprodutiva materna, hábitos maternos, morbidade e trabalho maternos, assistência ao parto e pré-natal (ver Tabela 1). As condições de nascimento da criança foram investigadas também através de questionário e complementadas com informações existentes no prontuário.

Tabela 1 - Variáveis incluídas no Subprojeto Perinatal. Pelotas, RS, 1993.

Situação socioeconômica

renda familiar

educação materna e paterna

tipo de trabalho do chefe da família

\author{
Condições demográficas \\ tamanho da família \\ idade materna \\ idade paterna \\ número de filhos \\ idade dos filhos
}

\section{Condições de gravidez}

patologias ocorridas durante a gestação

utilização dos serviços de pré-natal

época de início de consulta

número de consultas

qualidade do atendimento

hábitos maternos durante a gestação

trabalho materno

\author{
Condições de parto \\ tipo de parto \\ motivo para cesariana (se for o caso) \\ pessoa que realizou o parto
}

\section{Condições ambientais}

condições de saneamento

suprimento de água

condições de moradia

\author{
Condições reprodutivas prévias \\ abortos anteriores \\ natimorto \\ RN pré-termo \\ RN baixo peso \\ intervalo interpartal
}

\author{
Condições do RN \\ gênero \\ idade gestacional \\ peso ao nascer \\ comprimento e perímetro cefálico \\ contagem de Apgar \\ morbidade perinatal
}

\section{Morbimortalidade materna \\ morbidade puerperal \\ mortalidade materna}


As maternidades eram visitadas diariamente. Todos os recém-nascidos foram pesados ao nascer com balanças pediátricas de mesa com precisão de $10 \mathrm{~g}$, aferidas semanalmente com pesos-padrão. Os recém-nascidos eram também examinados pela equipe de pesquisa. O comprimento era medido na posição supina com material padronizado (infantômetros AHRTAG) ${ }^{2}$ e o perímetro cefálico com fita métrica inelástica, ambos com precisão de $1 \mathrm{~mm}$. A idade gestacional foi avaliada através do método de Dubowitz ${ }^{5}$ realizado sistematicamente em todos os recém-nascidos no primeiro dia de vida. O exame consiste na avaliação de critérios neurológicos e somáticos do recém-nascido que são pontuados de acordo com escala previamente estabelecida.

Em relação à antropometria materna, o peso do início da gravidez era colhido através do questionário baseado na informação da mãe ou do cartão da gestante quando disponível. As mães foram pesadas usando balança de uso doméstico e medidas usando antropômetro desenvolvido pela equipe da pesquisa. A cor da mãe era observada pelo entrevistador e registrada no questionário.

O controle de qualidade dos dados foi garantido através de diversos mecanismos ${ }^{15}$. Os questionários foram ampla e repetidamente pré-testados e eram acompanhados de um manual de instruções detalhando as perguntas e orientando sobre como proceder em caso de dúvida. Os mesmos cuidados na preparação de questionários e manuais foram tomados para todos os demais subprojetos.

A equipe do Subprojeto Perinatal era composta de um coordenador $(\mathrm{RH})$, dois pediatras e sete acadêmicos de Medicina, previamente treinados durante sete dias. Em dezembro de 1992, foi realizado um estudo piloto. Durante o ano, cada entrevistador examinou crianças em todas as cinco maternidades. Este rodízio serviu para evitar que os vícios pessoais de certos entrevistadores viessem a comprometer os dados coletados em um determinado hospital. Para que nenhum hospital ficasse a descoberto, havia dois entrevistadores suplentes treinados, à disposição para qualquer substituição necessária. O controle de qualidade da informação obtida era reforçado através de reuniões regulares com todos os entrevistadores. Uma amostra aleatória de cerca de 5\% das entrevistas e avaliações da idade gestacional foram repetidas por dois outros pediatras, com a finalidade de verificar a qualidade e veracidade dos dados. Durante todo o estudo, não se encontrou nenhuma entrevista forjada, e o índice kappa ${ }^{6}$ para o escore de Dubowitz entre os entrevistadores e a revisita foi 0,71 , o que garante a qualidade da informação.

Tabela 2 - Variáveis incluídas no Subprojeto de Acompanhamento. Pelotas, RS, 1993-94.

\section{Situação socioeconômica \\ renda familiar \\ trabalho materno}

\section{Antropometria \\ peso \\ comprimento \\ perímetro cefálico \\ circunferência abdominal}

\section{Morbidade referida}

diarréia

infecções respiratórias

cólicas

acidentes

\section{Dieta}

amamentação e causas de desmame recordatório alimentar de 24 horas idade de introdução dos alimentos

\section{Outros}

uso de chupetas

número de acidentes

padrões de choro

\section{Condições ambientais}

características da moradia

suprimento de água e saneamento

exposição à fumaça do tabaco

\section{Cuidados da criança}

freqüência à creche

quem cuida da criança

treinamento esfincteriano

atividades da criança

papel do pai nos cuidados

\section{Uso de serviços}

hospitalizações

consultas médicas e não médicas

imunizações

monitorização do crescimento

acesso a serviços de saúde

consumo de medicamentos 


\section{Metodologia do Subprojeto de Acompanhamento}

A fim de avaliar a evolução das crianças durante o primeiro ano de vida, subamostras dos nascidos vivos urbanos foram acompanhados aos 1, 3, 6 e 12 meses de idade (Figura 1). Os principais grupos de variáveis estudadas constam da Tabela 2.

No primeiro e terceiro mês tentou-se acompanhar 655 crianças. Este subprojeto teve como objetivo principal descrever os hábitos alimentares, crescimento e morbidade nos primeiros meses de vida. Especial destaque foi dado para o estudo do uso de chupetas na causação do desmame precoce. A equipe era composta de um epidemiologista (EW) e três entrevistadoras.

Para o acompanhamento do sexto e décimo segundo mês, tentou-se localizar todas as crianças com peso de nascimento inferior a $2.500 \mathrm{~g}$, assim como uma amostra de $20 \%$ das demais crianças, incluindo todas aquelas visitadas no acompanhamento do primeiro e terceiro mês. A razão para incluir todas as crianças de baixo peso foi o desejo de estudar em detalhes sua morbidade e crescimento. Esta equipe foi coordenada por um epidemiologista $(\mathrm{BH})$ e uma nutricionista (PRVH), contando ainda com cinco entrevistadores.

As crianças a serem incluídas na amostra foram escolhidas através de amostragem sistemática ${ }^{15}$. Ao se completar cada mês do estudo perinatal, todas as crianças eram listadas por ordem de dia do nascimento, sendo escolhida uma quota mensal de aproximadamente 55 crianças para o acompanhamento do primeiro e terceiro

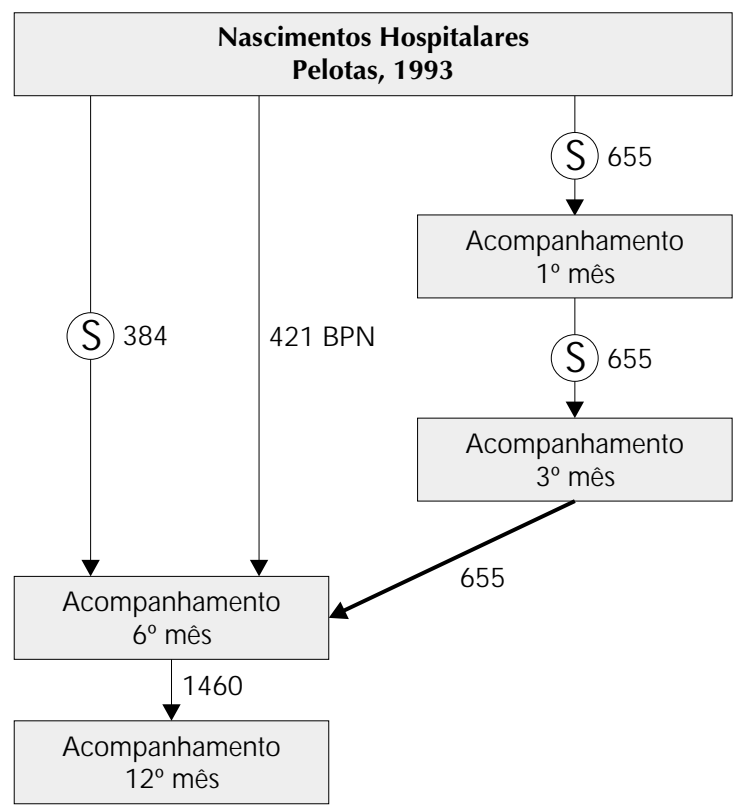

Figura 1 - Seleção de sujeitos mês. Para o acompanhamento do sexto e décimo segundo mês, além de todas as crianças mencionadas e daquelas com baixo peso ao nascer, eram sorteadas sistematicamente 32 crianças adicionais por mês, em média.

O subprojeto iniciou em fevereiro de 1993 - quando a primeira criança completou um mês - e terminou ao final de dezembro de 1994 - quando a última completou um ano de vida. Todas as crianças eram pesadas com balanças portáteis modelo $\mathrm{CMS}^{15}$ com precisão de 100 $\mathrm{g}$, aferidas semanalmente com pesos-padrão. O comprimento era medido na posição supina com material padronizado (infantômetros AHRTAG) ${ }^{15}$ e os perímetros cefálico e abdominal com fita métrica inelástica. Todas as medidas tinham precisão de $1 \mathrm{~mm}$.

A localização dos endereços em um estudo de coorte é um aspecto crítico. Uma série de medidas foram tomadas para tentar minimizar as perdas. Durante o estudo perinatal, eram coletadas, de todas as mães, informações detalhadas referentes ao endereço atual e como encontrá-lo (linha e parada de ônibus, referências locais), local de trabalho do pai e mãe, endereço de parentes próximos, telefones para contato, e - se a família pretendia mudar-se em breve - endereço futuro. A Tabela 3 mostra as perdas e recusas ocorridas durante o estudo. Mesmo aos 12 meses de idade, o percentual de perdas foi reduzido $(6,6 \%)$.

Nos acompanhamentos de um e três meses, respectivamente, 99,2\% e 98,6\% das entrevistas foram realizadas dentro do mês de aniversário das crianças, sendo estes percentuais, para os acompanhamentos de seis e doze meses, $94,7 \%$ e $97,5 \%$, respectivamente.

Os acompanhamentos foram realizados por equipes de entrevistadores das área de Nutrição, Enfermagem e Medicina. Estes foram treinados durante uma semana em técnicas de entrevista e de antropometria. Amostras aleatórias de $10 \%$ das entrevistas foram repetidas por supervisores, utilizando uma versão condensada dos questionários para evitar tomar tempo demasiado das mães.

\section{Subprojeto de Mortalidade}

Foram estudados todos os óbitos fetais tardios (ocorridos a partir de 28 semanas de gestação), neonatais precoces (durante a primeira semana de vida) e pósneonatais precoces (demais óbitos ocorridos durante o primeiro ano de vida, isto é, entre os 7 e 364 dias completos). Como de praxe, a mortalidade perinatal incluiu óbitos fetais tardios e neonatais precoces, enquanto que a mortalidade infantil incluiu os óbitos neonatais precoces acrescentados aos demais óbitos ocorridos no primeiro ano. Este subprojeto propiciou descrever os níveis e causas de mortalidade, assim com seus principais determinantes.

A equipe do subprojeto era composta por uma epidemiologista (AMM), três pediatras, dois patologistas e uma entrevistadora. Os óbitos foram monitorizados através de visitas diárias aos quatro principais hospitais 
da cidade, e de visitas semanais ao quinto hospital que apresentava um reduzido número de internações pediátricas. Em cada um dos quatro hospitais maiores, as maternidades e Unidades de Tratamento Intensivo eram visitadas diariamente, enquanto que os berçários, enfermarias pediátricas e serviços de Pronto Socorro onde raramente ocorriam óbitos - eram visitados quinzenalmente. Para identificar óbitos ocorridos fora dos hospitais, eram realizadas visitas mensais aos cartórios, cemitérios e à Delegacia Regional de Saúde.

A coleta de dados estendeu-se de primeiro de janeiro de 1993 a 31 de dezembro de 1994, quando a criança médica domiciliar com a mãe da criança falecida, quando se coletava uma história clínica completa dos eventos que precederam o óbito, baseada numa adaptação dos questionários utilizados na Investigação Inter-Americana de Mortalidade na Infância ${ }^{14}$. Não houve nenhuma recusa para essas entrevistas.

Tentou-se de todas as maneiras completar as informações acima através de necrópsias com a exceção dos natimortos para os quais a necrópsia é pouco reveledora ${ }^{13}$. Obtida a autorização por escrito dos pais, a solicitação era feita pelo pediatra que atendeu ao óbito, sendo as necrópsias realizadas diariamente (inclusive

Tabela 3 - Números de crianças selecionadas, de recusas e perdas no Subprojeto de Acompanhamento. Pelotas, RS, 1993-94

\begin{tabular}{lcccc}
\hline Acompanhamento (mês) & Amostra original & Recusas & Endereço não localizado & Percentual entrevistado \\
\hline 1 & 655 & 2 & 4 & $99,1 \%$ \\
3 & 655 & 2 & 9 & $98,3 \%$ \\
6 & 1.460 & 8 & 38 & $96,8 \%$ \\
12 & 1.460 & 11 & 85 & $93,4 \%$ \\
\hline
\end{tabular}

mais jovem de coorte completou um ano. Dos 166 óbitos (incluindo os fetais tardios) ocorridos neste período, 21 foram domiciliares, quatro ocorreram no berçário, três nas enfermarias e o restante nas Unidades de Tratamento Intensivo. Foi possível entrevistar os médicos assistentes e familiares de todos estes óbitos, com uma única exceção de um óbito domiciliar cujo endereço não pôde ser localizado.

\section{Investigação da causa de óbito}

Para detectar a causa básica do óbito fetal e o momento em que ocorreu (ante ou intrapartum), o obstetra era entrevistado pela epidemiologista encarregada do estudo, descrevendo o parto e opinando sobre a causa básica da morte. Perguntava-se especificamente sobre a ocorrência das seguintes complicações: descolamento de placenta, prolapso de cordão, trabalho de parto prematuro espontâneo, apresentação pélvica, placenta prévia, eclâmpsia, incompetência de colo uterino, ruptura uterina, doenças infecciosas pélvicas, hipertensão arterial sistêmica e outras doenças. Nesta oportunidade, confirmava-se com o obstetra a idade gestacional do feto morto.

Para os óbitos infantis a entrevista era realizada com o pediatra. Ainda era feita uma revisão sistemática do prontuário, no caso de crianças hospitalizadas, em busca das seguintes informações: motivo da internação, história da doença atual, exame clínico na admissão, evolução, tratamento, exames laboratoriais e diagnóstico. Tanto para os óbitos fetais como para os neonatais precoces utilizavam-se ainda as informações do questionário perinatal aplicado à mãe logo após o nascimento.

Para os óbitos ocorridos entre os 7 e 364 dias, estas informações eram complementadas por uma entrevista finais de semana) no Laboratório de Patologia da Faculdade de Medicina. O transporte de corpo era feito gratuitamente pela funerária da Prefeitura Municipal. Apesar de todos esses esforços, o percentual de necrópsias atingido foi de apenas $31,7 \%$ para os óbitos neonatais precoces. Entre os 49 óbitos ocorridos, entre os 7 e 364 dias, não se logrou obter autorização dos pais para nenhuma necrópsia, embora cinco destes tenham sido autopsiados no Instituto Médico Legal por exigência da legislação (óbitos domiciliares sem assistência médica).

A determinação da causa básica da morte era feita por dois médicos independentes, baseados nas informações dos prontuários hospitalares, das entrevistas com obstetras e pediatras, dos dados obtidos com as mães e quando existentes, dos resultados das necrópsias. Cada um dos árbitros preenchia um atestado de óbito para cada criança. Havendo discordância, um terceiro árbitro, também médico pediatra, tomava a decisão final. O coordenador da Divisão de Informação em Saúde da Secretaria da Saúde e do Meio Ambiente do Rio Grande do Sul realizou a codificação desses atestados conforme a $9^{\mathrm{a}}$ versão da Classificação Internacional de Doenças ${ }^{12}$.

\section{Estudo Aninhado de Casos e Controles para Óbitos entre os 7 e 364 Dias}

Para melhor entendimento dos fatores de risco associados a mortalidade entre os 7 e 364 dias, delineouse um estudo de casos e controles aninhado na coorte de nascimentos. Este estudo excluiu os óbitos perinatais porque as informações sobre possíveis fatores de risco já estavam disponíveis no questionário aplicado por ocasião do nascimento. 
Para cada óbito detectado eram selecionadas duas crianças-controle nascidas no mesmo dia, imediatamente antes e após o nascimento do caso. No domicílio, as mães de casos e controles eram então entrevistadas a fim de avaliar fatores de risco tais como dieta, moradia, cuidados da criança e uso de serviços. As entrevistas eram realizadas por uma entrevistadora não médica. Dos 98 controles selecionados, dois não puderam ser localizados, sendo substituídos pelos próximos nascimentos.

\section{Subprojeto de Morbidade Hospitalar}

Para medir a incidência de hospitalizações no primeiro ano de vida e indicar suas principais causas, foram monitorizadas todas as admissões hospitalares de crianças urbanas nascidas em 1993. A hospitalização era definida como a permanência em ambiente hospitalar por um período igual ou superior a $24 \mathrm{~h}$ Internações no período perinatal foram caracterizadas como permanência de $24 \mathrm{~h}$ ou mais após o parto, devido à patologia apresentada pela criança. A permanência no pós-parto imediato em Pelotas rarissimamente excede as $24 \mathrm{~h}$, a não ser em caso de cesariana. Estes casos, assim como quando a criança permanecia no hospital em decorrência de doença da mãe, não eram incluídos no Subprojeto de Morbidade Hospitalar.

De janeiro de 1993 até dezembro de 1994, os quatros principais hospitais da cidade foram visitados diariamente. O quinto hospital, com reduzido número de internações, era visitado a cada semana. Em cada hospital eram visitadas as enfermarias, serviço de Pronto Socorro, berçários, Unidades de Tratamento Intensivo e quartos particulares. A equipe do subprojeto foi composta por um médico epidemiologista (JAC), três pediatras e seis entrevistadores (estudantes de Medicina matriculados a partir do sexto semestre). Os entrevistadores faziam rodízio trimestral entre os diversos hospitais. Um total de 1.314 hospitalizações foram identificadas durante o estudo, sendo que 952 crianças foram hospitalizadas.

Dentro dos hospitais, os entrevistadores revisavam os prontuários para todas as crianças. Não foi possível localizar 24 dos 1.376 prontuários $(1,7 \%)$ por motivo de extravio, sendo estas hospitalizações excluídas do estudo. Quando o prontuário era pouco informativo, realizavase também uma entrevista com o médico pediatra. Com exceção das crianças que haviam permanecido no hospital imediatamente após o nascimento, entrevistavamse as mães sobre os sinais e sintomas observados antes da hospitalização. Praticamente todas as entrevistas foram realizadas dentro do hospital, mas em menos de $1 \%$ dos casos foi necessário visitar os domicílios para colher estes dados. Não houve nenhuma recusa e somente $1,7 \%$ de perdas nesta fase. Para 8,6\% dos prontuários não foi possível estabelecer a causa básica da internação em virtude das poucas informações disponíveis nos hospitais.

A causa da internação era determinada inicialmente por dois árbitros (pediatras) independentes, com base nas informações do prontuário (anamnese, exame físico, exames complementares, tratamento e evolução), da entrevista com as mães e, quando cabível, da entrevista com o pediatra. Esses árbitros, trabalhando separadamente, tentavam estabelecer a causa básica da internação. Em caso de discordância, um terceiro árbitro revisava os dados existentes e emitia seu parecer. Quando este concordava com um dos dois árbitros iniciais, o diagnóstico ficava estabelecido. Em caso de nova discordância, os três árbitros se reuniam e chegavam a um diagnóstico de consenso. Os diagnósticos eram codificados conforme a Classificação Internacional de Doenças $^{12}$.

\section{Estudo Aninhado de Casos e Controles para Morbidade Hospitalar}

Para investigar os fatores de risco para as internações por doenças infecciosas ocorridas após o primeiro mês de vida, realizou-se um estudo de casos e controles aninhados na coorte. Através de visita domiciliar, aplicava-se às mães dos casos hospitalares o mesmo questionário usado no estudo de acompanhamento. Para as crianças internadas com idade entre 30 e 89 dias, usava-se o questionário do acompanhamento de um mês de vida. Para as crianças hospitalizadas com 90 a 179 e 180 a 364 dias, aplicavam-se respectivamente os questionários dos acompanhamentos dos três e seis meses. As informações obtidas para os casos eram referidas para a data base do acompanhamento (isto é, 30, 90 e 180 dias de idade). Esta comparação era restrita à primeira internação de cada criança, sendo excluídas as reinternações. De 164 casos ocorridos entre 30 e 89 dias houve quatro perdas $(2,5 \%)$ por mudança de endereço; de 211 ocorridos entre 90 e 189 dias houve três perdas $(1,5 \%)$ e das restantes 246,11 perdas $(5 \%)$.

O grupo-controle era composto de todas as crianças incluídas nos acompanhamentos. Quando o próprio caso já havia sido entrevistado por pertencer à subamostra do estudo de acompanhamento, não era necessário realizar uma nova entrevista. No entanto, estas últimas crianças permaneciam também no grupo-controle, o que caracteriza o estudo como um delineamento do tipo casobase ou inclusivo ${ }^{11}$.

\section{Subprojeto de Desenvolvimento Psicomotor}

O diagnóstico precoce de atrasos no desenvolvimento infantil é essencial para melhorar o prognóstico das crianças afetadas ${ }^{10}$. No entanto, os desvios do desenvolvimento em lactentes são de difícil identificação no exame pediátrico de rotina, sendo necessários testes específicos. O subprojeto do desenvolvimento infantil teve como objetivo descrever os padrões de desenvolvimento infantil durante o primeiro ano de vida; avaliar o valor preditivo do escore de desenvolvimento no primeiro, terceiro e sexto mês de vida em relação ao escore dos doze meses; e verificar as possíveis 
associações entre atrasos na área motora, linguagem e social com fatores socioeconômicos, características perinatais e aleitamento materno.

A metodologia incluiu o uso do Teste de Denver $\mathrm{II}^{8}$, o instrumento mais amplamente utilizado por pediatras para triagem dos problemas de desenvolvimento ${ }^{7}$. Embora não padronizado para o Brasil, estudos em outros países mostraram variações geográficas reduzidas, especialmente na área de linguagem e mesmo assim apenas em crianças maiores de um ano.

Os entrevistadores dos estudos de acompanhamento de 1, 3, 6 e 12 meses foram treinados na administração do teste, utilizando material padronizado, e $5 \%$ das testagens foram repetidas para garantir a qualidade da informação. Quando eram evidenciadas alterações na testagem estas crianças eram encaminhadas ao serviço de saúde.

\section{Aspectos Éticos}

A investigação foi aprovada pelo Comitê Científico da Faculdade de Medicina em seus aspectos técnicos e éticos. A permissão para realizar a entrevista era solicitada a todas as mães, e a confidencialidade dos dados coletados era garantida. Crianças ou mães que, durante o estudo de acompanhamento, apresentavam algum problema de saúde eram encaminhadas aos serviços médicos correspondentes.

\section{Procesamento de Dados}

Os questionários utilizados nos diferentes subprojetos eram compostos quase que exclusivamente de perguntas fechadas, cuja codificação era realizada pelos entrevistadores. As eventuais perguntas abertas eram codificadas posteriormente por dois supervisores que também revisavam a qualidade geral do questionário. Os entrevistadores de todos os subprojetos receberam treinamento específico sobre codificação, incluindo a necesssidade de clareza na grafia dos algarismos, peculiaridades da codificação de cada pergunta, instruções sobre perguntas abertas, entre outros.

A digitação do questionário perinatal era feita no máximo cinco dias após o nascimento da criança. Este procedimento possibilitou a rapidez nos sorteios das subamostras dos acompanhamentos de 1, 3, 6 e 12 meses. Os questionários coletados para estas subamostras eram também digitados imediatamente.

O processamento incluiu a criação de arquivos de entrada de dados através do pacote estatístico EpiInfo $5.01^{4}$. O primeiro a ser trabalhado foi o arquivo para os registros do Estudo Perinatal, constituído de aproximadamente 280 variáveis, para as quais foi preparado um programa específico de verificação da amplitude e consistência das variáveis coletadas.

Ao longo do estudo, realizaram-se várias limpezas dos dados coletados até então. Obtinham-se freqüências das variáveis coletadas para identificar e corrigir inconsistências de codificação, revisão e digitação. Ao término da entrada de dados, foram obtidas novamente frequiências de todas as variáveis para a edição final.

$\mathrm{Na}$ medida em que ocorriam óbitos de crianças, esta informação era incorporada semanalmente ao arquivo principal. Isto tinha a função de evitar que os óbitos fossem sorteados para os acompanhamentos das subamostras.

Os bancos de dados de todos os subestudos foram preparados de maneira a permitir sua posterior combinação. Todos os questionários tinham uma variável de identificação comum, a qual recebia o mesmo nome nos diversos arquivos. Variáveis similares (por exemplo, peso com 1, 3, 6 e 12 meses) recebiam nomes diferentes mas consistentes (por exemplo, peso1, peso3, etc.). A Tabela 4 mostra o número de casos e variáveis em cada estudo.

\section{RESULTADOS}

Através das visitas hospitalares, foram identificados 6.410 nascimentos em 1993. Destes, 42 crianças haviam nascido em casa, sendo levadas para o hospital e incluídas no estudo. Das crianças incluídas, $5.304(82,9 \%)$ nasceram de mães residentes na zona urbana, $434(6,8 \%)$ da zona rural e $658(10,3 \%)$ de outras localidades. Ao final do acompanhamento perinatal foram contabilizadas sete perdas e nove recusas, ou seja, $0,3 \%$ do total. Houve 115 partos gemelares correspondendo a $1,8 \%$ do total.

Em virtude da dificuldade logística de acompanhar os nascimentos de zona rural e de outras localidades, os subprojetos de acompanhamento foram restritos à população urbana da cidade. Os resultados abaixo se referem a esta população. A presente publicação inclui os primeiros resultados do estudo, sendo os resultados de cada subprojeto objetos de publicações subseqüentes.

A Tabela 5 mostra a distribuição da amostra conforme características familiares e maternas. Revelou-se uma população pobre, com cerca de uma em cinco crianças nascendo em famílias com renda igual ou inferior a um salário-mínimo (US\$70). As mães tinham em média 6,7 anos de escolaridade, e uma em cada oito estava já separada do pai da criança. Dezessete por cento das mães eram adolescentes e uma em cada três era primípara. Apenas uma mãe em cada vinte não havia tido nenhuma assistência pré-natal, sendo que mais de $80 \%$ tiveram cinco ou mais consultas. A altura média era de cerca de $160 \mathrm{~cm}$ e o ganho médio de peso durante a gestação de $11,6 \mathrm{~kg}$. Um terço das mães eram fumantes e quatro quintos foram caracterizadas como de cor branca.

$\mathrm{O}$ peso de nascimento continua sendo o fator de risco isolado mais importante na determinação da 
Tabela 4 - Número de crianças e de variáveis incluídas em cada base de dados. Pelotas, RS, 1993-94.

\begin{tabular}{lcc}
\hline Estudo & Número de crianças & Número de variáveis \\
\hline Perinatal & 5.304 & 401 \\
1 mês & 649 & 188 \\
3 meses & 644 & 233 \\
6 meses & 1.414 & 339 \\
12 meses & 1.343 & 275 \\
M ortalidade & $264^{\mathrm{a}}$ & 160 \\
M orbidade & 952 & 96 \\
\hline Total & - & 1.692 \\
\hline a & &
\end{tabular}

a 166 casos e 98 controles

morbimortalidade infantil ${ }^{1}$. A Tabela 6 mostra a distribuição de peso ao nascer para todos os nascimentos urbanos assim como após exclusão de crianças de partos múltiplos e natimortos. Para os recém-nascidos urbanos após a exclusão de crianças de partos múltiplos e de natimortos, o peso médio ao nascer foi de $3.169 \mathrm{~g}$ (d.p. $538 \mathrm{~g}$ ) e houve $9,1 \%$ de baixo peso ao nascer $(<2.500 \mathrm{~g}), 8,9 \%$ de retardo de crescimento intra-uterino e $8,0 \%$ de nascimentos pré-termo. A Figura 2 mostra a combinação desses diversos grupos.

Foram detectados 117 óbitos perinatais durante o estudo, sendo 55 no período fetal e 62 no período neonatal precoce. Dentre os óbitos fetais, 39 ocorreram antes do trabalho de parto e 16 durante o mesmo. Dos óbitos neonatais precoces, $53 \%$ ocorreram no primeiro dia e $47 \%$ entre o segundo e o sétimo dia.

Estudos longitudinais permitem o cálculo exato das probabilidades de morte na coorte. Para fins de comparação com a literatura, no entanto, estes são aqui referidos como "coeficientes de mortalidade".

O coeficiente de mortalidade fetal foi de 10,5 por 1.000 nascimentos, de mortalidade neonatal precoce de 11,7 por 1.000 e o de mortalidade perinatal de 22,1 por 1.000 nascimentos. Houve ainda 49 óbitos infantis pós-perinatais, dos quais 13 ocorreram no período entre o $8^{\circ}$ e o $28^{\circ}$ dia de vida. Com os 62 óbitos neonatais precoces, foi computado o total de 111 óbitos infantis. O coeficiente de mortalidade infantil foi de 21,1 para 1.000 nascimentos.

Dois óbitos fetais, um óbito neonatal precoce e dois óbitos pós-neonatais não foram incluídos na análise. Quatro deles por ter a mãe recusado a entrevista perinatal e um deles por ter sido impossível localizar o endereço.

No estudo de morbidade hospitalar as principais causas de hospitalizações no primeiro ano de vida estão descritas na Tabela 7, de acordo com a Classificação Internacional de Doenças ${ }^{12}$. As afecções do período perinatal foram responsáveis por $34,0 \%$ das internações, seguidas pela pneumonia com $16,1 \%$ e as doenças diarréicas com 14,6\%.

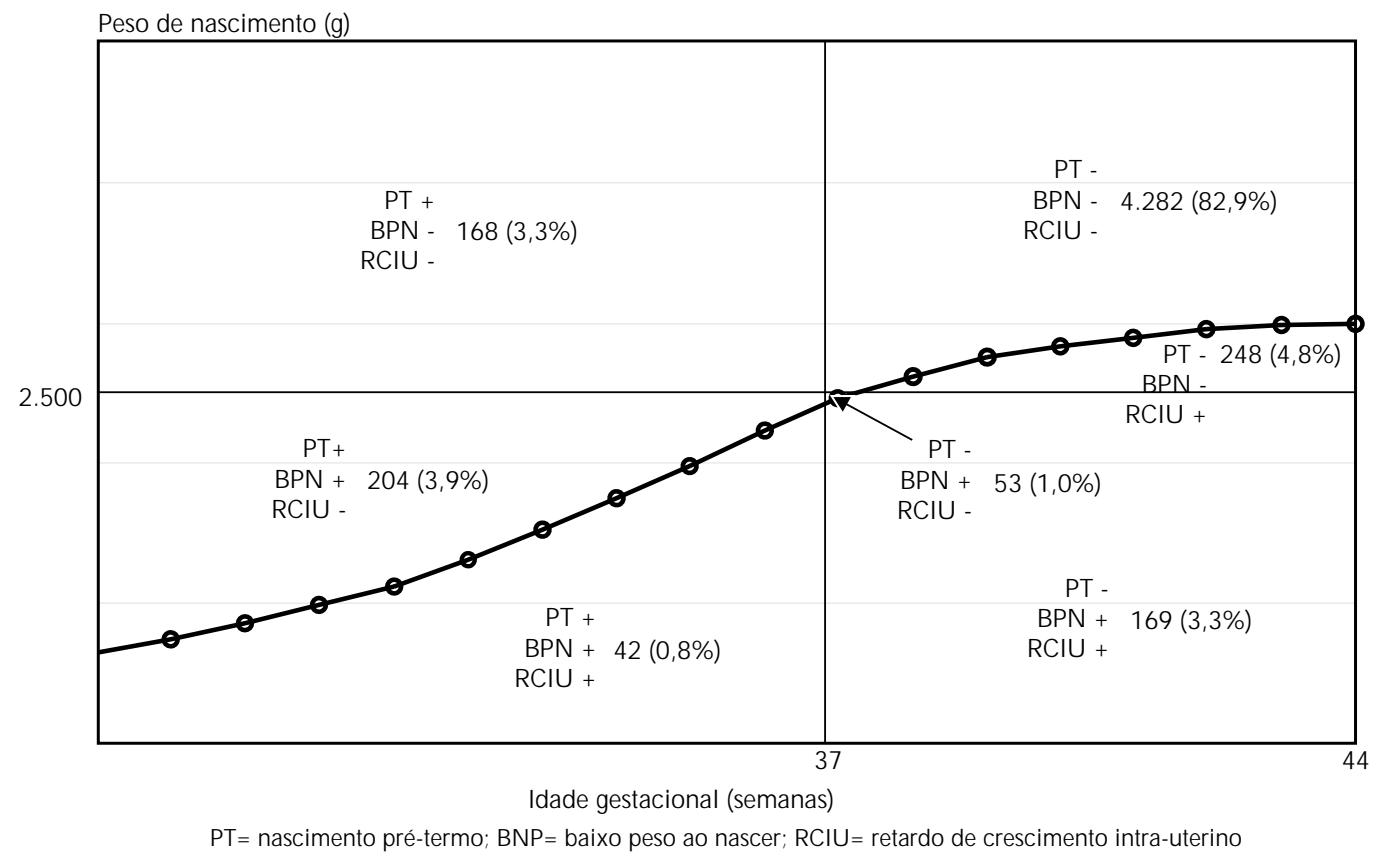

Figura 2 - Distribuição das crianças de acordo com o peso de nascimento, idade gestancional e crescimento intrauterino. Pelotas, RS, 1993 
Tabela 5 - Distribuição dos nascimentos urbanos conforme características maternas. Pelotas, RS, 1993.

\begin{tabular}{cr}
\hline Características maternas & Percentual ou média \\
\hline Renda familiar (salários-mínimos) & \\
$\leq 1$ SM & $19,0 \%$ \\
$1.1-3$ SM & $41,7 \%$ \\
$3.1-6$ SM & $23,5 \%$ \\
$6.1-10$ SM & $8,4 \%$ \\
$>10$ SM & $7,4 \%$
\end{tabular}

Escolaridade (anos completos)

$\begin{array}{rr}\text { média } & 6,7 \text { anos } \\ \%<3 \text { anos } & 9,3 \%\end{array}$

Sem companheiro

$12,3 \%$

Idade

$\begin{array}{rr}\text { média } & 26,0 \text { anos } \\ \%<20 \text { anos } & 17,4 \%\end{array}$

Paridade

$\begin{array}{rr}\text { média } & 1,8 \text { nascimentos } \\ \% \text { de primíparas } & 35,1 \%\end{array}$

História reprodutiva prévia aborto(s)

natimorto(s)

nascido(s) com baixo peso cesariana(s)

Atenção pré-natal (consultas)

$\begin{array}{rr}0 & 4,9 \% \\ 1-4 & 13,0 \% \\ 5-9 & 53,9 \% \\ \text { mais } & 28,3 \%\end{array}$

Altura materna

$\begin{array}{rr}\text { média } & 159,8 \mathrm{~cm} \\ \%<150 \mathrm{~cm} & 4,6 \%\end{array}$

Peso no início da gestação

média

$\%<50 \mathrm{~kg}$

$58,2 \mathrm{~kg}$

$15,7 \%$

Peso no final da gestação

média

$\%<55 \mathrm{~kg}$

$69,9 \mathrm{~kg}$

$6,8 \%$

Ganho de peso na gestação

$$
\begin{array}{r}
\text { média } \\
\%<9 \mathrm{~kg}
\end{array}
$$

$11,6 \mathrm{~kg}$

$29,3 \%$

Fumantes $(\%)$

$33,5 \%$

Cor branca (\%)

$77,2 \%$

Número de nascimentos
Tabela 6 - Distribuição de peso ao nascer para os recémnascidos urbanos. Pelotas, RS, 1993.

\begin{tabular}{lrr}
\hline Peso ao nascer & \multicolumn{2}{c}{ Número (\%) } \\
\cline { 2 - 3 } & $\begin{array}{c}\text { N ascimentos } \\
\text { totais }\end{array}$ & $\begin{array}{c}\text { N ascidos vivos } \\
\text { de parto único }\end{array}$ \\
\hline$<1.000 \mathrm{~g}$ & $21(0,4 \%)$ & $11(0,2 \%)$ \\
$1.000-1.499 \mathrm{~g}$ & $34(0,6 \%)$ & $24(0,5 \%)$ \\
$1.500-1.999 \mathrm{~g}$ & $90(1,7 \%)$ & $82(1,6 \%)$ \\
$2.000-2.499 \mathrm{~g}$ & $380(7,2 \%)$ & $351(6,8 \%)$ \\
$2.500-2.999 \mathrm{~g}$ & $1.312(24,7 \%)$ & $1.283(24,9 \%)$ \\
$3.000-3.499 \mathrm{~g}$ & $2.057(38,8 \%)$ & $2.039(39,6 \%)$ \\
$3.500-3.999 \mathrm{~g}$ & $1.083(20,4 \%)$ & $1.079(21,0 \%)$ \\
$>4.000 \mathrm{~g}$ & $281(5,3 \%)$ & $280(5,4 \%)$ \\
$\mathrm{N}$ ão Pesados & $46(0,9 \%)$ & - \\
\hline Total & $5.304(100 \%)$ & $5.166(100 \%)$ \\
\hline
\end{tabular}

Tabela 7 - Percentuais de crianças hospitalizadas conforme a causa. Pelotas, RS, 1993.

\begin{tabular}{lcc}
\hline Causa de internação & No de crianças & Percentual \\
\hline Causas perinatais & 308 & $34,0 \%$ \\
Pneumonia & 140 & $14,7 \%$ \\
Diarréia & 123 & $13,3 \%$ \\
O utras causas respiratórias & 81 & $8,6 \%$ \\
Bronquiolite & 82 & $8,6 \%$ \\
Demais causas & 218 & $20,8 \%$ \\
\hline Total & 952 & $100,0 \%$ \\
\hline
\end{tabular}

Nos acompanhamentos de 1, 3, 6 e 12 meses, os percentuais de crianças amamentadas foram respectivamente $86,4 \%, 58,6 \%, 36,9 \%$ e $23,1 \%$, evidenciando uma curta duração do aleitamento materno.

A prevalência de déficit de crescimento de acordo com os índices de peso/idade, comprimento/ idade e peso/comprimento está descrita na Tabela 8. Nos diversos acompanhamentos houve déficit de crescimento mais marcados ( -2 d.p. NCHS) nas relações comprimento/idade e peso/idade do que para o peso/comprimento.

\section{DISCUSSÃO}

O presente tipo de estudo é inédito no Brasil: duas coortes de nascimentos, ambas de base populacional, separadas por um período de onze anos. O alto percentual de partos hospitalares - em 1982 menos de $1 \%$ dos partos foram realizados no domicílio $^{1}$ - garante a representatividade das mães estudadas nos hospitais para a população da cidade. O único estudo semelhante, de nosso conhecimento, foram as coortes inglesas de nascimentos de $1958 \mathrm{e}$ $1970^{3}$. Outros aspectos metodológicos do estudo merecem ser salientados. Os altos índices de 
Tabela 8 - Prevalência de déficits antropométricos em diferentes idades. Pelotas, RS, 1993*.

\begin{tabular}{lcccc}
\hline Indicador & \multicolumn{4}{c}{ Estudo de acompanhamento (idade) } \\
\cline { 2 - 4 } & 1 mês & 3 meses & 6 meses & 12 meses \\
\hline Peso/idade & $3,4 \%$ & $1,1 \%$ & $1,9 \%$ & $3,8 \%$ \\
Comprimento/idade & $6,0 \%$ & $4,9 \%$ & $4,3 \%$ & $6,1 \%$ \\
Peso/comprimento & $0,5 \%$ & $0,8 \%$ & $0,2 \%$ & $0,9 \%$ \\
\hline * Abaixo de -2 desvios- padrão em relação à referência do NCHS. & &
\end{tabular}

* Abaixo de -2 desvios- padrão em relação à referência do NCHS.

seguimento das crianças ( $93,4 \%$ aos 12 meses) são pouco usuais em estudos de coorte, particularmente quando realizados em países em desenvolvimento. A utilização do método de Dubowitz e sua alta repetibilidade, expressa através do índice kappa, é também inovadora em estudos epidemiológicos. De nosso conhecimento, parece ser este o maior estudo já ocorrido no mundo, utilizando este método de avaliação precisa da idade gestacional.

Outro aspecto a ser salientado é a realização de dois estudos de casos e controles aninhados na coorte, um sobre mortalidade infantil e o segundo sobre as hospitalizações. Este delineamento utiliza as vantagens do estudo de coorte, evitando os viéses de seleção - que consistem na principal limitação dos estudos de casos e controles - assim como faz uso da maior eficiência desses estudos, evitando os custos de entrevistar todos os não-casos.

$\mathrm{O}$ estudo evidenciou uma população em plena transição epidemiológica. Os coeficientes de mortalidade perinatal e infantil apresentaram queda de $30 \%$ a $50 \%$ em relação a 1982 (de 32,2 para 22,1 por 1.000 nascimentos, e de 36,4 para 21,1 , por 1.000 nascimentos, respectivamente). No entanto, a freqüência de baixo peso ao nascer mostrou-se praticamente inalterada nesta década. A prevalência de déficit de comprimento/idade aos 12 meses revelou-se estável $(5,5 \%$ para $6,1 \%)$, embora houvesse uma redução nos déficits de peso/idade (de $5,4 \%$ para $3,8 \%$ ). A duração da amamentação aumentou de 3,1 meses em 1982 para 4,0 meses em 1993. Em resumo, apesar de importantes quedas na mortalidade, observou-se que outros indicadores de saúde, como o peso ao nascer e o déficit de crescimento linear mantiveram-se estáveis. Estes resultados podem ser interpretados como revelando melhoria no atendimento médico à população materno-infantil, embora as condições básicas de saúde e nutrição do feto e do lactente tenham-se mantido inalteradas. Esses tópicos serão alvos de publicações futuras.

Alguns resultados do presente estudo estão sendo de grande utilidade para o sistema local de saúde, principalmente no planejamento das ações das unidades periféricas. Através do endereço residencial fornecido pela mãe, os nascimentos foram alocados às diferentes unidades da cidade. Com isto, foi possível medir indicadores básicos para a populaçãoalvo de cada posto de saúde, como a proporção de baixo peso ao nascer, de partos por cesarianas e de freqüência ao pré-natal, o estado nutricional das crianças, a amamentação e a cobertura vacinal, entre outros. Além disso, informações dos bancos de dados foram repassadas para a equipe de planejamento da Secretaria Municipal de Saúde.

Os hospitais envolvidos no estudo receberam o retorno das informações referentes aos nascimentos ali ocorridos, assim como os indicadores globais para toda a cidade. Este retorno propiciou reflexões sobre o atendimento oferecido ao parto e ao recémnascido nos hospitais de Pelotas, servindo como referencial para melhorias nos serviços de saúde. Os resultados do estudo estão também sendo retornados para a população geral através de uma série de artigos na imprensa local.

Grande número das informações coletadas das crianças e suas mães ainda necessitam ser analisadas. Cada subprojeto deverá gerar resultados específicos e mais detalhados que permitirão compreender melhor as transições ocorridas nos últimos onze anos e seus determinantes.

\section{AGRADECIMENTOS}

Aos Hospitais da cidade de Pelotas, na pessoa de seus diretores na área de Pediatria, Ginecologia e Obstetrícia Drs.: Amilcare A. Vecchi, Danilo R. de Moura, José A. Granzoto, Lígia Iara da Silveira, Maria Conceição de O. Fernandez, Marco Antônio S. Funchal, Miguel L. Piltcher, Renato R. Riemke, Roberto V. Gazalle, Silvio S. Reis, pela ampla colaboração na realização da etapa perinatal do estudo. Ao Dr. Paulo Grassi da Secretaria de Saúde do Rio Grande do Sul, e aos patologistas Dr. Flávio Menezes e Dr. Heitor Janke pela sua indispensável colaboração ao subprojeto de mortalidade. Ao Delegado de Saúde Dr. Luís Felipe Ustarroz, pela sua colaboração no acesso aos atestados de óbitos. 
Finalmente, a todos os bolsistas que participaram ativamente de todas as fases deste projeto, e que foram fundamentais para o sucesso na coleta e preparo dos dados:

Estudo Perinatal: Andréa Bertoni, Bernardo Sobreiro, Carlo Endrigo Nunes, Tanira Pires Freitas, Ana Luiza Curi Hallal, Edilson Silva Machado, Benilde Rosa Polo, Tatiana Vincenci.

Estudo Mortalidade: Cristina da Rocha, Clarita Pereira Alves.

Estudo Morbidade Hospitalar: Adelar Breitenbach, Mônica Hammes Stone, Osmar Bonacina, Vânia Fracalocci, Fernando Ramos.

\section{REFERÊNCIAS BIBLIOGRÁFICAS}

1. BARROS, F.C; VICTORA, C.G.; VAUGHAN, J.P. The Pelotas birth cohort study, 1982-1987: strategies for following up 6,000 children in a developing country. Perinatal Pediatr. Epidemiol., 4:267-82, 1990.

2. BARROS, F.C \& VICTORA, C.G. Epidemiologia da saúde infantil: um manual para diagnósticos comunitários. São Paulo, UNICEF/Hucitec, 1991.

3. CHAMBERLAIN, R.; CHAMBERLAIN, G.; HOWLETT, B.; CLAREAUX, A. British births 1970. London, Heinemann Medical Books, 1975. v. 1: The first week of life.

4. DEAN, A.G.; DEAN, J.A.; BURTON, A.H.; DICKER R.C. Epi-Info, Version 5: a word processing, database and statistics programme for epidemiology on micro-computers. Atlanta, Center for Disease Control, 1990.

5. DUBOWITZ, L.M.S.; DUBOWITZ, V. GOLDBERG, C. Clinical assessment of gestational age in the newborn infant. J. Pediatr., 77: 1-10, 1970.

6. FLEISS, J.F. Statistical methods for rates and proportions. 2nd ed. New York, Wiley, 1981.

7. FRANKENBURG, W.K. \& DODDS, J.B. The Denver developmental screening test. Denver, Co., University of Colorado Medical Center, 1969.

8. FRANKENBURG, W.K. \& DODDS, J.B. The Denver II developmental screening test. Denver, Co. Denver Developmental Materials, 1992.
Estudo Perinatal e de Morbidade Hospitalar: Elaine Pinto Albernaz, Luciani Oliveira e Simone Karam.

Acompanhamentos 1 e 3 meses: Eliana Fernandes Barbosa, Neuza Harter, Patrícia Moura, Márcia Vaz, Marco Antônio Almeida.

Acompanhamentos 6 a 12 meses: Lúcia Berndt, Renata Maciel, Cleusa Amaral, Luciana Silva, Milene Sedrez, Juares Bassani.

Digitação e limpeza de dados: Maria Angélica Neves, Carmen Moreira, Jefferson Padilha, Evandro Mulinari, Liriane Comerlato, Cristiano Moreira, Edi Marlene Santana Monteiro.

9. MACEDO, R. Adjustment with a human face. In: Cornia G.A. et al. The state of the world's children. Oxford University Press, 1987. Cap. 2.

10. MEISELS, J. S. \& SHONKOFF, J. P. ed. Handbook of early childhood intervention. Oxford, 1990.

11. LAST, J. M. A dictionary of epidemiology. New York, Oxford University Press, 1983

12. ORGANIZAÇÃO MUNDIAL DA SAÚDE. Classificação Internacional de Doenças: revisão 1975. São Paulo, Centro da OMS para Classificação de Doenças em Português, 1980.

13. POTTER, E.L. \& GRAIG, J. M. Postmortem examination. In: Potter, E.L. \& Graig, J.M. Pathology of the fetus and the infant. $3^{\text {rd }}$ ed. Chicago, Year Book Medical Publishers, 1975. p. 91 .

14. PUFFER, R. R. \& SERRANO, C. V. Patterns of mortality in childhood: the inter-american investigation of mortality in childhood.Washington, DC PAHO, 1973 (Scientific Publication n. 262).

15. VICTORA, C.G.; BARROS, F. C.; VAUGHAN, J.P. Epidemiologia da desigualdade: um estudo longitudinal de 6.000 crianças brasileiras. $2^{\mathrm{a}}$ ed. São Paulo, CEBES/ Hucitec, 1989. 